\title{
Existence of thought force and its characteristics
}

\section{Dhananjay Pal}

Pharmacy College, Bengal School of Technology, Sugandha-Delhi Road, Chuchura, Dist.-Hooghly, , West Bengal, INDIA.

\author{
Email address: \\ dhananjay.pal123@gmail.com,paldhananjay46@yahoo.com
}

\section{To cite this article:}

Dhananjay Pal. Existence of Thought Force and Its Characteristics. American Journal of Physical Chemistry.

Vol. 2, No. 5, 2013, pp. 94-104. doi: 10.11648/j.ajpc.20130205.13

\begin{abstract}
A single field emerged at the origin of the universe containing within itself the blueprint of the physical universe. The primordial single field triggered the onset of the universe. Most physicists believe that a single super-force dominated the first instants of creation. As per Penrose, consciousness is a part of the universe. Our consciousness model involving thought-carrying particle (TCP), thought retaining particle (TRP) and thought force $\left(\mathrm{T}_{\mathrm{F}}\right)$ signifies the existence of universal consciousness that exists along with the universe. This universal consciousness is a functional state of the universal mind. This universal mind (UM) is evolved at the Big Bang from Void. The UM is constituted by these TCP and TRP in the inherent presence of thought force $\left(T_{F}\right)$. Thought force $\left(T_{F}\right)$ is an expression of universal consciousness. This $T_{F}$ being the primordial quantum field functions as the original super-force. $\mathrm{T}_{\mathrm{F}}$ being the original super-force functions as the origin of all the fundamental fields. TCP is the carrier of thought force $\left(\mathrm{T}_{\mathrm{F}}\right)$ that, in turn, appears to be the origin of all the fields. The quantized energy $\left(\varepsilon_{T}\right)$ of TCP is responsible to cause the cosmic microwave background radiation temperature as well as the universal consciousness. The individual consciousness owes its origin to the universal consciousness. The same $\varepsilon_{T}$ is the energy responsible for generating thought force $\left(\mathrm{T}_{\mathrm{F}}\right)$. This $\mathrm{T}_{\mathrm{F}}$ being applicable to any inanimate object as well as to any biological system (having thinking ability) exerts its function both in vitro and in vivo. Existence of thought force and its characteristics are expressed here.
\end{abstract}

Keywords: Cosmic Microwave Background Radiation (CMBR), Thought Force ( $\left.\mathrm{T}_{\mathrm{F}}\right)$, Though-Carrying Particle (TCP), Universal Mind (UM), Thought Retaining Particle (TRP), Quantized Energy $\left(\varepsilon_{T}\right)$ of TCP

\section{Introduction}

In Eastern philosophical traditions, consciousness is intrinsic to the universe, whereas in most Western views, consciousness is extrinsic, emerging from complex computation. How can these views be reconciled?

1.1. In contrast to the usual linear sequence of matter, body, life, brain, mind, consciousness, here the proposed cyclic sequence is first universal consciousness (a functional state of the universal mind), and then matter, body, life, brain, and regeneration of mind and consciousness. The evolution of life with mind and consciousness is possible purely due to the inherent existence of universal consciousness which exists along with the universe. The human nervous system is evolved to provide an appropriate material structure to individualize the universal consciousness, a characteristic of reality, pervading all manifestations.

As per Penrose [1], consciousness is a part of the universe. Van De Bogart [2] explained, "Since consciousness is a part of the universe it then follows that all consciousness, and the universe, are of the same matrix of energy fields".

It is most relevant and important to indicate the names of various eminent physicists like Erwin Schrödinger, Eugene Wigner, Brian Josephson, John Wheeler, Roger Penrose, Henry P Stapp, Freeman J. Dyson, Paul Davies, David Bohm, Basil Hiley, Fritjof Capra, Fred Alan Wolf and Amit Goswami who have addressed the inclusion of consciousness in their work. Consciousness is to be taken into account.

Consciousness model of Pal et al [3-5] involving TCP, TRP and thought force $\left(\mathrm{T}_{\mathrm{F}}\right)$ signifies the existence of universal consciousness that exists along with the universe. The universal consciousness is a functional state of the universal mind (UM). We showed the existence of thought force $\left(\mathrm{T}_{\mathrm{F}}\right)$ that, in turn, is an expression of the universal consciousness.

Bhaumik [6] mentioned, "It would be reasonable to presume that the universe originated in a unity of all fields at or near Planck's dimension. John Wheeler strongly believes that "in defining any useful concept of reality" we have to take into account "the indispensable place of the 
participating observer---evidenced in quantum mechanics," the foregoing cannot be really true unless consciousness is as essential as aspect of nature as are the fields that give rise to force and matter and the primary field that gave rise to them".

\section{Evolution of Universe}

\section{General view for the evolution of the universe}

Big Bang $\rightarrow$ Universe $\rightarrow$ [Fields + Particles \& Antiparticles + STC $] \rightarrow[$ Matter and Fields + STC $] \rightarrow$ Matter and Fields + STC + Life as well as consciousness.

Many physicists agree with the idea that consciousness is non-local and fundamental in the universe. Scientists have arrived at a simple but decisive conclusion that consciousness is very much a part of the universe, like other objects. As per Roger Penrose [1], consciousness is a part of the universe. If the universe exists along with the universal consciousness that, in turn, is a functional state of the universal mind (UM) and if this UM is evolved at the Big Bang from the eternal void, then

\section{Our view for the evolution of the universe (see Scheme-I) is as follows}

VOID $\rightarrow$ [Big Bang] $\rightarrow$ [TCP, TRP \& Anti-TRP in the inherent presence of thought force $\left.\left(\mathrm{T}_{\mathrm{F}}\right)+\mathrm{STC}\right] \rightarrow$ [Universal Mind with universal consciousness + TCP \& TRP in the inherent presence of thought force $\left(\mathrm{T}_{\mathrm{F}}\right)+\mathrm{STC}+$ $\mathrm{UTF}] \rightarrow$ [Universal Mind with universal consciousness + Matter and Fields \{including thought force $\left(\mathrm{T}_{\mathrm{F}}\right)$ \} + TCP \& $\mathrm{TRP}+\mathrm{STC}+\mathrm{UTF}] \rightarrow$ Universal Mind with universal consciousness + Matter and Fields \{including thought force $\left.\left(\mathrm{T}_{\mathrm{F}}\right)\right\}+$ Life as well as consciousness $+\mathrm{TCP} \& \mathrm{TRP}+\mathrm{STC}$ + UTF [where thought force $\left(\mathrm{T}_{\mathrm{F}}\right)$ is the origin of all the fields, TCP (Though-carrying particle) is the origin of all the field particles, TRP (Thought retaining particle) is the origin of all the matter particles, anti-TRP is the origin of all the anti-particles, STC $=$ Space time continuum and $\mathrm{UTF}=$ Universal thought frequency $\cong$ frequency of the TCP].

Physicists determined that underlying quantum fields give birth to elementary particles. Bhaumik [6] mentioned that Frank Wilczek pointed out, "In quantum field theory, the primary elements of reality are not individual particles, but underlying fields. Thus, for example, all electrons are but excitations of an underlying field, naturally called electric field". The same holds true for all the fundamental particles of which matter is made.

The existence of matter depends on the existence of force and vice versa. Matter particles are usually designated as "Fermions", because they follow Fermi-Dirac Statistics; they are of spin $1 / 2$ or its multiple. Matter particles obey Pauli's exclusion principle. Force particles are usually designated as "Bosons", because they follow Bose-Einstein Statistics; they are of spin 0, or any integer like 1, 2, 3 etc. Force particles do not obey Pauli's exclusion principle.

TCP cannot exist without TRP and vice versa. Many physicists believe that unifying all the forces, including gravity, into a single theory would require a phenomenon called super-symmetry. With super-symmetry, every fermion would have a boson twin, and vice-versa. TCP that behaves like boson should accompany its supersymmetrical partner TRP that functions like fermion in the generalized simpler way. Thus TCP like boson cannot have anti-particle. But TRP that functions like fermion should have its anti-particle and here it is shown as Anti-TRP (see Scheme-I). It is to be noted that these TCP and TRP function like wavicle: wave-particle duality.

2.1. As per the ancient Vedanta, there is only one Infinite Existence called BRAHMAN. This BRAHMAN is more or less equivalent to the Void of modern science. This Void is not the meaning of nothingness. This Void is the source of infinite energy. Anything and everything of this universe are its manifestations. Hawking [7] mentioned, "The Heisenberg's uncertainty principle established that the Void is filled with infinite pairs of virtual particles and antiparticles. These pairs would have an infinite amount of energy and therefore, by Einstein's equation: $\mathrm{E}=\mathrm{mc}^{2}$, they would have an infinite amount of mass". Without the uncertainty principle to forbid nothingness, there might not even be a universe.

Ranganathananda [8] quoted the following:

\section{The UPANISADS say (Chândogya Upanisad, IV.10.4)}

"Brahman is life. Brahman is joy.

Brahman is the Void-

Joy, verily, that is the same as the Void.

The Void, verily, that is the same as Joy".

Pal et al [5] explained that the eternal 'Void' has been covered by the mind to function as the Soul of individual being. In the universe, behind the Universal Mind (UM), there is a Soul ( $\cong$ VOID). In the individual, behind the individual mind (which is a constituent of the UM), there is also a Soul ( $\cong$ VOID).

Capra [9] expressed through the 'vacuum diagram' that the 'Void' is the source of all the matters prevailing in this universe. Capra [9] explained this fact through a 'vacuum diagram' as shown below:

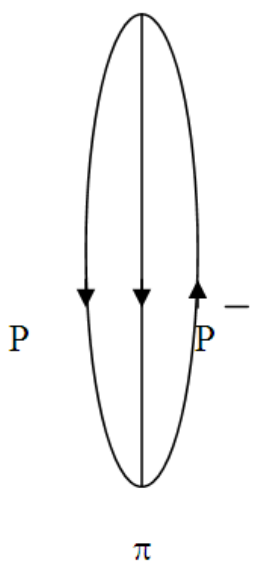

\section{A Vacuum diagram}

This Vacuum diagram explains the process: three particles---a proton $(\mathrm{P})$, an antiproton $\left(\mathrm{P}^{-}\right)$, and a pion $(\pi)$ are formed out of nothing and disappear again into the vacuum. According to the field theory, events of that kind 
happen all the time. The vacuum is far from empty. On the contrary, it contains an unlimited number of particles, which come into being and vanish without end.

Similar to the 'vacuum diagram' as explained by Capra
[9], the evolution of the universe from the eternal 'Void' can also be lucidly explained through the generation of postulated TRP, anti-TRP and TCP as shown below:
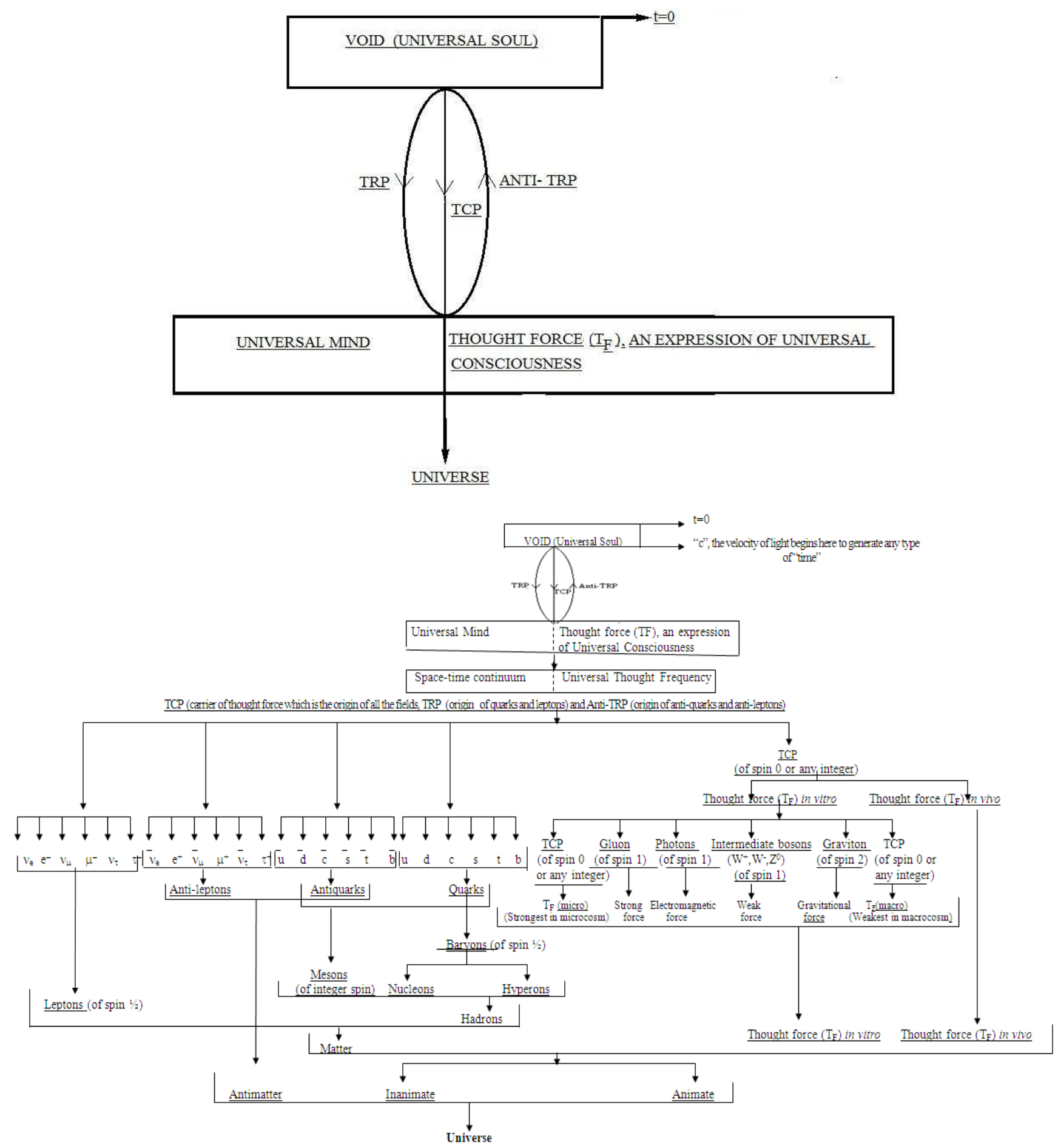

Scheme-I: Schematic presentation showing the evolution of the universe from the Void incorporating the postulated TCP (Thought-carrying particle), TRP (Thought-retaining particle) and Thought Force $\left(T_{F}\right)$. Laws of absolute conservation of mass, charge (and color) are to be maintained in order to develop a general theory for the unification of physics which would be freely applicable to the more general situations involving both the non-living system and living system having consciousness. Here TCP is the carrier of thought force $\left(T_{F}\right)$ that is the origin of all the fields. TRP is the origin of all the matter particles. It is to be noted that these TCP and TRP function like wavicle: wave-particle duality. Here thought force (TF) is an expression of the universal consciousness 


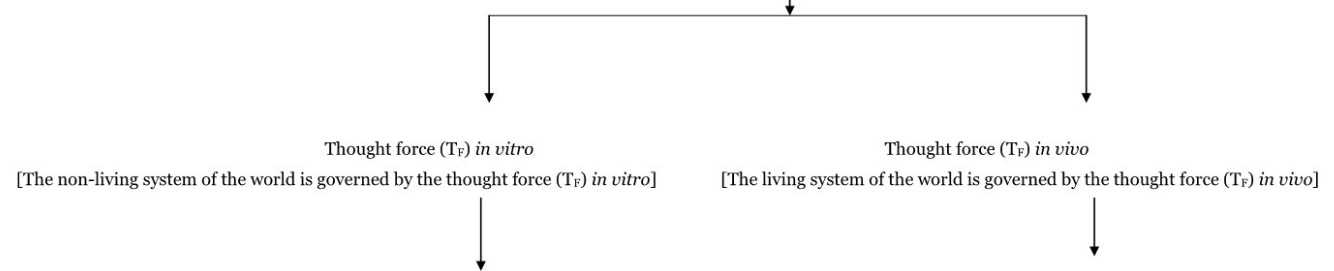

$\mathrm{T}_{\mathrm{F}}$ (micro), SNF, EMF, WNF, GF and $\mathrm{T}_{\mathrm{F}}$ (macro)

where $\mathrm{T}_{\mathrm{F}}(\mathrm{micro})=$ Thought force in microcosm, $\mathrm{SNF}=$ Strong nuclear force,

$\mathrm{EMF}=$ Electromagnetic force, $\mathrm{WNF}=$ Weak nuclear force,

$\mathrm{GF}=$ Gravitational force and $\mathrm{T}_{\mathrm{F}}$ (macro) $=$ Thought force in macrocosm.

It is expressed here that $\mathrm{T}_{\mathrm{F}}$ (micro) is a stronger force than the SNF and

$T_{\mathrm{F}}$ (macro) is a weaker force even than the GF.

\begin{abstract}
Thought force $\left(\mathrm{T}_{\mathrm{F}}\right)$ in vivo is a type of force that represents the biological 'thought' which is the action of mind. This 'thought' being a type of force controls the 'thought processes' involving the firing of neurons through the quantum mechanical activities of these postulated TCP and TRP in the presence of consciousness. This consciousness, in turn, is the quantized energy $\left(\varepsilon_{\tau}\right)$ of the TCP. Several experiments signify the existence of thought force $\left(\mathrm{T}_{\mathrm{F}}\right)$ in vivo.
\end{abstract}

Scheme-II: Thought force and its significant roles: Most physicists believe that a single super-force dominated the first instants of creation. This mysterious super-force is the postulated Thought force $\left(\mathrm{T}_{\mathrm{F}}\right)$. It is possible that the evolution of all the fundamental fields is originated from this super-force that is the Thought force $\left(\mathrm{T}_{\mathrm{F}}\right)$ in our view. This thought force $\left(\mathrm{T}_{\mathrm{F}}\right)$ is carried by the TCP (thought-carrying particle) in the presence of its super-symmetrical partner TRP (though retaining particle). TCP is the carrier of thought force $\left(T_{F}\right)$ that, in turn, is the origin of all the fields. TCP is the origin of all the field particles. TRP is the origin of all the matter particles. TCP canno exist without TRP and vice versa. Our consciousness model involving TCP, TRP and thought force $\left(\mathrm{T}_{\mathrm{F}}\right)$ signifies the existence of universal consciousness that exists along with the universe. We showed that the quantized energy $\left(\varepsilon_{T}\right)$ of the TCP is responsible to cause the universal consciousness as well as the cosmic microwave background radiation temperature. The individual consciousness owe its origin to the universal consciousness created by the same $\varepsilon_{T}$. The same $\varepsilon_{T}$ is the energy responsible for generating Thought force $\left(\mathrm{T}_{\mathrm{F}}\right)$. Thus, Thought force $\left(\mathrm{T}_{\mathrm{F}}\right)$ is an expression of the universal consciousness. Thought force $\left(\mathrm{T}_{\mathrm{F}}\right)$ being an expression of the universal consciousness is applicable to any inanimate object as well as to any biological system (having thinking ability). The Thought force $\left(\mathrm{T}_{\mathrm{F}}\right)$ exerts its functions both in vitro and in vivo. The non-living system of the world is governed by the thought force $\left(\mathrm{T}_{\mathrm{F}}\right)$ in vitro and this Thought force $\left(\mathrm{T}_{\mathrm{F}}\right)$ in vitro gives rise to $\mathrm{T}_{\mathrm{F}}(\mathrm{micro}), \mathrm{SNF}, \mathrm{EMF}$ $\mathrm{WNF}, \mathrm{GF}$ and $\mathrm{T}_{\mathrm{F}}$ (macro) where $\mathrm{T}_{\mathrm{F}}($ micro $)=$ Thought force in microcosm, $\mathrm{SNF}=$ Strong nuclear force, $\mathrm{EMF}=$ Electromagnetic force, $\mathrm{WNF}=$ Weak nuclear force, $\mathrm{GF}=$ Gravitational force and $\mathrm{T}_{\mathrm{F}}$ $($ macro $)=$ Thought force in macrocosm. It is to be noted here that $\mathrm{T}_{\mathrm{F}}$ (micro) is a stronger force tha the $\mathrm{SNF}$ and $\mathrm{T}_{\mathrm{F}}$ (macro) is a weaker force even than the GF. The living system of the world is governed by the thought force $\left(\mathrm{T}_{\mathrm{F}}\right)$ in vivo and this Thought force $\left(\mathrm{T}_{\mathrm{F}}\right)$ in vivo is a type of force that

Schematic presentation showing the evolution of Universe from the 'Void' (see Scheme-I).

This Scheme lucidly shows the evolution of Universe from the Void incorporating the postulated TCP (Thoughtcarrying particle), TRP (Thought-retaining particle) and Thought Force $\left(\mathrm{T}_{\mathrm{F}}\right)$. Laws of absolute conservation of mass, charge (and color) are to be maintained in order to develop a general theory for the unification of physics which would be freely applicable to the more general situations involving both the inanimate and animate having consciousness.

2.2. Pal et al $[3,5]$ showed that the functional state of Universal Mind (UM) is the universal consciousness that exists along with the universe. This UM is evolved at the Big Bang from the eternal Void (see Scheme-I). This Void, in turn, is the source of infinite energy. And this UM is a finer matter. The individual mind being a constituent of the UM is also a finer matter. The constituents of the UM and individual mind are same. The ultimate constituents of matter and mind are same as both mind and matter are aspects of one fundamental reality, which is called UM. The brain is the mediating link or interface between the individual mind and the body. The constituents of UM are the ultimate constituents of matter itself as everything in this universe is a manifestation of this UM.

Pal et al $[3,5]$ showed that the UM is constituted by represents the biological 'thought' which is the action of mind. This 'thought' being a type of force controls the 'thought processes' involving the firing of neurons through the quantum mechanical activities of these postulated TCP and TRP in the presence of consciousness. This consciousness, in turn, is the quantized energy $\left(\varepsilon_{T}\right)$ of the TCP. Thought force $\left(\mathrm{T}_{\mathrm{F}}\right)$ being the primordial quantum field functions as the original single primary unified field that is not only the origin of all the four fundamental fields along with both the $\mathrm{T}_{\mathrm{F}}$ (micro) and $\mathrm{T}_{\mathrm{F}}$ (macro) but also the origin of Thought force $\left(\mathrm{T}_{\mathrm{F}}\right)$ in vivo.

Note: What was previously called a force is now usually called a field or interaction. The existence of matter depends on the existence of force and vice versa. Matter particles are usually designated as "Fermions", because they follow Fermi-Dirac Statistics. Matter particles obey Pauli's exclusion principle; they are of spin $1 / 2$. . Force particles are usually designated as "Bosons", because they follow Bose-Einstein Statistics. Force particles do not obey Pauli's exclusion principle; they are of spin 0 , or any integer like 1, 2, 3 etc. TCP cannot exist without TRP and vice versa. Many physicists believe that unifying all the forces, including gravity, into a single theory would require a phenomenon called super-symmetry. With super-symmetry, every fermion would have a boson twin, and vice-versa. TCP that behaves like boson should accompany its super-symmetrical partner TRP that functions like fermion in the generalized simpler way.

these TCP and TRP in the inherent presence of thought force $\left(T_{F}\right)$. As the UM is constituted by these TCP and TRP, so the ultimate constituents of matter and mind are these TCP and TRP in the inherent presence of thought force $\left(\mathrm{T}_{\mathrm{F}}\right)$ in vitro and thought force $\left(\mathrm{T}_{\mathrm{F}}\right)$ in vivo [See Scheme-I].

2.3. Pal et al [5] developed three different equations expressing the quantized energy $\left(\boldsymbol{\varepsilon}_{T}\right)$ of TCP radiated from the radiant mass of universe. The value of $\varepsilon_{T}$ in one of the three equations is shown below:

$$
\varepsilon_{T}
$$

$=4.384 \times 10^{-16} \mathrm{erg} \equiv 2.73 \times 10^{-4} \mathrm{eV} \equiv 2.73^{0} \mathrm{~K} \cong C M B R$ temperature $\equiv 2.725^{\circ} \mathrm{K}$

It is indicated by Weisskopf [10] that $1 \mathrm{erg} \cong 0.6241807 \times 10^{12} \mathrm{eV}$ and $10^{-4} \mathrm{eV} \cong 1^{0} \mathrm{~K}$ where $\mathrm{K}=$ Kelvin.

2.4. Pal et al [5] expressed that the CMBR temperature is due to $\varepsilon_{T}$, the quantized energy of TCP where $\varepsilon_{T}=4.384 \times 10^{-16} \mathrm{erg} \equiv 2.73^{\mathrm{o}} \mathrm{K}$ that is very close to $2.725^{\circ} \mathrm{K}$ (CMBR temperature). As per the proposition of Pal et al [5], these TCP, TRP and Thought force $\left(\mathrm{T}_{\mathrm{F}}\right)$ had played their significant roles at the onset of the universe and they exist till today in this universe. For this reason, Pal et al [5] wanted to find out any possible relationship 
between the CMBR temperature and quantized energy of the proposed TCP. Three different equations expressing the quantized energy ( $\varepsilon_{T}$ ) of TCP have been shown by Pal et al [5] and it is interesting to note that all of them ultimately give rise to the same result that is equivalent to the CMBR temperature. A sort of relationship is thus observed between the $\varepsilon_{T}$ and CMBR temperature. This coincidence is thus signifying a probable role of TCP on the maintenance of CMBR temperature. Further, this coincidence is also signifying the existence of these TCP in the presence of TRP. TCP cannot exist without TRP and vice versa. The presence of TCP, TRP and Thought force $\left(\mathrm{T}_{\mathrm{F}}\right)$ in the universe is thus indicated and expressed mathematically. At present we are unable to explain when and how these TCP and TRP decoupled from the primordial cosmic soup.

\section{Thought Force and Its Characteristics}

The natural forces that the modern scientists usually encounter in this world are of four types:

(1) Gravitational force (GF) (mediated by graviton of spin 2), (2) Electromagnetic force (EMF) (mediated by photon of spin 1), (3) Weak nuclear force (WNF) (mediated by $\mathrm{W}^{+}, \mathrm{W}^{-}, \mathrm{Z}^{0}$ of spin 1), (4) Strong nuclear force (SNF) (mediated by eight kinds of gluons of spin 1).

Weinberg [11], Perkins [12] and Quigg [13] reported that there would have to exist a new class of 'extra strong' interaction which would be stronger than the existing strong nuclear force (SNF) along with the existence of a new class of 'long-range weakest' force which would be weaker even than the Gravitational force (GF).

Consciousness model of Pal et al [3,5] indicates that the universe exists along with the universal consciousness that, in turn, is created by the quantized energy $\left(\varepsilon_{T}\right)$ of TCP. This $\varepsilon_{T}$ of TCP exists along with the universe to cause the universal consciousness as well as the CMBR temperature.

Pal et al $[3,5]$ explained that the quantized energy $\left(\varepsilon_{T}\right)$ of TCP is the energy responsible for generating thought force $\left(T_{F}\right)$; thus the $T_{F}$ may be expressed as

$$
T_{F}=\varepsilon_{T} / D_{i}
$$

where $D_{i}=$ Interacting distance.

The thought force $\left(\mathrm{T}_{\mathrm{F}}\right)$ has been postulated to be carried by TCP in the inherent presence of its super-symmetrical partner TRP. The TCP being the carrier of $\mathrm{T}_{\mathrm{F}}$ would behave like bosons when TRP would function like fermions.

Depending on the $D_{i}$ (= Interacting distance), we can calculate and identify two new forces viz. $\mathrm{T}_{\mathrm{F}}$ (micro) $[=$ Thought force in microcosm] and $\mathrm{T}_{\mathrm{F}}$ (macro) [= Thought force in macrocosm], the existence of which is indicated and expressed here.

A single field emerged at the origin of the universe containing within itself the blueprint of the physical universe. The primordial single field triggered the onset of the universe. Most physicists believe that a single superforce dominated the first instants of creation. This mysterious super-force is the Thought force $\left(\mathrm{T}_{\mathrm{F}}\right)$ itself. It is possible that the evolution of all the fundamental fields is originated from this super-force that is the Thought force $\left(\mathrm{T}_{\mathrm{F}}\right)$ in our view.

Thought force $\left(\mathrm{T}_{\mathrm{F}}\right)$, an expression of the universal consciousness, is the primordial quantum field that, in turn, functions as the primary unified field. $\mathrm{T}_{\mathrm{F}}$ itself may thus be found to be the original single primary unified field that is the origin of all the four fundamental fields along with both the $\mathrm{T}_{\mathrm{F}}$ (micro) and $\mathrm{T}_{\mathrm{F}}$ (macro). This $\mathrm{T}_{\mathrm{F}}$ being an expression of the universal consciousness is applicable to any inanimate object as well as to any biological system (having thinking ability). Thus the $\mathrm{T}_{\mathrm{F}}$ being an expression of the quantized energy $\left(\varepsilon_{T}\right)$ of TCP exerts its functions both in vitro and in vivo (see Scheme-II).

In a purpose to involve both the non-living and living systems of the world, we have shown the existences of these TCP, TRP and thought force $\left(\mathrm{T}_{\mathrm{F}}\right)$ in vitro and thought force $\left(\mathrm{T}_{\mathrm{F}}\right)$ in vivo. Any one can call this TCP by any other name, but as the highly developed living system will have to be evolved in the universe in the long run and as the thought of highly developed living system appears to be a kind of force to be called the thought force $\left(\mathrm{T}_{\mathrm{F}}\right)$ in vivo, we considered it is wise to call it as TCP. Further, as the universe exists along with the universal consciousness that, in turn, is created by the quantized energy $\left(\varepsilon_{T}\right)$ of TCP, we had to use the term TCP.

The non-living system of the world is governed by the thought force $\left(\mathrm{T}_{\mathrm{F}}\right)$ in vitro and this

Thought force $\left(\mathrm{T}_{\mathrm{F}}\right)$ in vitro gives rise to $\mathrm{T}_{\mathrm{F}}$ (micro), $\mathrm{SNF}$, EMF, WNF, GF and $\mathrm{T}_{\mathrm{F}}$ (macro)

where $\mathrm{T}_{\mathrm{F}}$ (micro) $=$ Thought force in microcosm, $\mathrm{SNF}=$ Strong nuclear force, $\mathrm{EMF}=$ Electromagnetic force, $\mathrm{WNF}=$ Weak nuclear force, $\mathrm{GF}=$ Gravitational force and $\mathrm{T}_{\mathrm{F}}$ (macro) $=$ Thought force in macrocosm. It is to be noted here that $\mathrm{T}_{\mathrm{F}}$ (micro) is a stronger force than the SNF and $\mathrm{T}_{\mathrm{F}}$ (macro) is a weaker force even than the GF.

The living system of the world is governed by the thought force $\left(\mathrm{T}_{\mathrm{F}}\right)$ in vivo and this

Thought force $\left(\mathrm{T}_{\mathrm{F}}\right)$ in vivo is a type of force that represents the biological 'thought' which is the action of mind. This 'thought' being a type of force controls the 'thought processes' involving the firing of neurons through the quantum mechanical activities of these TCP and TRP in the presence of consciousness. Consciousness in living organisms is a process which involves the quantum mechanical activities of these TCP and TRP, the ultimate constituents of any matter as well as any mind in the inherent presence of thought force $\left(\mathrm{T}_{\mathrm{F}}\right)$ in vitro and the thought force $\left(\mathrm{T}_{\mathrm{F}}\right)$ in vivo as indicated by Pal et al [5]. These TCP and TRP govern the activities of neurons (not the other way round). Neurons are simply the equipments used to generate consciousness and awareness. This consciousness, in turn, is the quantized energy $\left(\varepsilon_{T}\right)$ of TCP. 
As several leading scientists have commented the universe is so finely tuned to allowing the emergence of conscious entities that it looks more than mere coincidence.

Davies [14] expressed, "If the basic laws of the universe were just slightly different, life and everything we know would not exist. So why is the universe just right for life? Could the universe have fine-tuned itself in order to bring about life and consciousness? At first sight, the idea seems preposterous. How could the universe in the first split second, when its laws were still malleable, know about the emergence of life billions of years later'?

Barrow and Tipler [15] in their "The Anthropic Cosmological Principle 1986" mentioned the view point of Dyson who pointed out, "The more I examine the universe and the details of its architecture, the more evidence I find that the universe in some sense must have known we were coming". Physical laws clamor for life: the universe knew we were coming. Our universe is perfectly tailored for life.

All these statements simply signify the existence of universal consciousness that is the primary cause for the evolution of life. Without the inherent existence of universal consciousness, the inanimate matter itself cannot generate life and consciousness. These postulated TCP and TRP, the constituents of UM and the ultimate constituents of matter and mind are thus liable to be originated from the Void at the Big Bang to evolve the space-time continuum and UM along with the universal consciousness as indicated by Pal et al $[3,5]$. In this way the universe could 'know about' the emergence of life billions of years later. Thought force $\left(\mathrm{T}_{\mathrm{F}}\right)$, an expression of this universal consciousness, is thus the primordial quantum field (see Scheme-I and Scheme-II).

\subsection{Calculation of $T_{F}$ (Micro) (= Thought Force in Microcosm) and $T_{F}$ (Macro) (= Thought Force in Macrocosm)}

Gell-Mann [16] pointed out, "Since the size of a particle is typically about $10^{-13} \mathrm{~cm}$, the minimum reaction time is less than $\left(10^{-13} / 10^{10}=\right) 10^{-23} \mathrm{sec}$ for a particle moving at the speed of light. What we mean when we call the strong interaction "strong" is that even in that brief time the strong force (SNF) is powerful enough to cause a reaction to take place. Electromagnetic reactions, being 100 times weaker than strong reactions, take around 100 times longer, or typically $10^{-21} \mathrm{sec}$ ond. Processes involving the weak interaction, which is $10^{-14}$ times weaker than the strong interaction, commonly take about $10^{-9}$ second". The shorter the time required for a reaction to occur, the stronger the force.

Quigg [13] mentioned, "A unified theory suggests that quarks can change into leptons and vice versa. As in any gauge theory, such an interaction would be mediated by a force particle". It is to be noted that these TCP and TRP function like wavicle: wave-particle duality. Pal [5] expressed that both the TCP and TRP have the same quantized energy and they behave like biophotons in animals and like simple photons in inanimate objects.
It is found theoretically that the radius of a TCP or TRP within a nucleon $\left(r_{T n}\right)=8.28 \times 10^{-18} \mathrm{~cm}$.

When the typical size of a particle $\approx 10^{-13} \mathrm{~cm}$, then

the reaction time $\left(=10^{-13} / 10^{10}\right)=10^{-23} \quad \mathrm{sec}$ represents $1 \mathrm{SNF}$.

When the size of a TCP or TRP $=r_{T n} \approx 10^{-18} \mathrm{~cm}$, then the reaction time $\left(=10^{-18} / 10^{10}\right)=10^{-28} \mathrm{sec}$ represents $10^{5} \mathrm{SNF}$.

Hence, $\mathrm{T}_{\mathrm{F}}$ (within a nucleon in microcosm $)=10^{5} \mathrm{SNF}$ when $D_{i}$ (Interacting distance $)=r_{T n} \approx 10^{-18} \mathrm{~cm}$.

When

$$
\varepsilon_{T}=4.384 \times 10^{-16} \mathrm{erg}
$$

and $D_{i}=r_{T n}=8.28 \times 10^{-18} \mathrm{~cm}$ in microcosm, it is found from the Equation (1) that

$\mathrm{T}_{\mathrm{F}}($ within a nucleon in microcosm $)=\varepsilon_{T} / D_{i}=52.94$ dyne (within $10^{-18} \mathrm{~cm}$ ).

Thus $\mathrm{T}_{\mathrm{F}}$ (micro) $\equiv 52.94$ dyne $\cong 10^{5} \mathrm{SNF}$ where $\mathrm{T}_{\mathrm{F}}$ (micro) $=$ Thought force in microcosm.

Again, when $\varepsilon_{T}=4.384 \times 10^{-16} \mathrm{erg}$ and $D_{i}=R$ (radius of the present universe) $=1.482 \times 10^{29} \mathrm{~cm}$ in the macrocosm, it is found from the Equation (1) that

$\mathrm{T}_{\mathrm{F}}($ in macrocosm $)=\varepsilon_{T} / D_{i}=2.958 \times 10^{-45}$ dyne.

If 52.94 dyne relating to the $\mathrm{T}_{\mathrm{F}}$ (micro) gives rise to $10^{5} \mathrm{SNF}$, then $2.958 \times 10^{-45}$ dyne would give rise to $5.58 \times 10^{-42} \mathrm{SNF}$.

Thus

$$
\mathrm{T}_{\mathrm{F}}
$$

(macro) $\equiv 2.958 \times 10^{-45}$ dyne $\cong 5.58 \times 10^{-42} S N F$ where $\mathrm{T}_{\mathrm{F}}$ (macro) $=$ Thought force in macrocosm.

\section{2. $T_{F}($ Micro $)=$ Thought Force in Microcosm}

As gluons are the "photons" of strong interaction (SNF), so TCP are the "photons" of the hypothetical "strongest interaction" to be called $\mathrm{T}_{\mathrm{F}}$ (micro) concerning TRP as the pre-constituents of quarks and leptons when a separation of ' $\mathrm{r}$ ' between TRP during the formations of quarks or leptons is less than that of $2.8 \times 10^{-13} \mathrm{~cm}$ in the well-known Yukawa potential as indicated by Perkins [12]:

$$
V=g\left(\frac{e^{-n r}}{r}\right)
$$

It is found theoretically that the radius of a TCP or TRP within a nucleon $\left(r_{T n}\right)=8.28 \times 10^{-18} \mathrm{~cm}$.

Similarly, it is found that the radius of a TCP or TRP within an electron $\left(r_{T e}\right)=2.113 \times 10^{-16} \mathrm{~cm}$.

It appears from the Equation (2) that when $r=r_{T n}=8.82 \times 10^{-18} \mathrm{~cm}$ 
$r=r_{T e}=2.113 \times 10^{-16} \mathrm{~cm}<<2.8 \times 10^{-13} \mathrm{~cm}$, then there is the possibility of the manifestation of a 'strongest interaction' which is theoretically found to be $10^{3}$ to $10^{5}$ times SNF in the realm of microcosm. This 'extra strong' interaction is the thought force in microcosm to be called $\mathrm{T}_{\mathrm{F}}$ (micro). Thus within a region of $10^{-18} \mathrm{~cm}$ to $10^{-16} \mathrm{~cm}$, this $\mathrm{T}_{\mathrm{F}}$ (micro) is the 'strongest force'.

These characteristics of $\mathrm{T}_{\mathrm{F}}$ (micro) indicate the existence of an "extra strong" interaction, often called "Technicolor" that would confine quarks and leptons within about $10^{-17}$ $\mathrm{cm}$ and which would reveal at energies of about $1 T e V\left(10^{12} \mathrm{eV}\right)$ as pointed out by Quigg [13] and which is theoretically found to be true by accepting the existence of these TCP and TRP.

$\mathrm{T}_{\mathrm{F}}$ (micro) is theoretically found to be the strongest interaction (a new class of 'extra strong' interaction). It is stronger than SNF. It is found that $T_{\mathrm{F}}$ (micro) $\cong 10^{5} \mathrm{SNF}$. This interaction is conjectured to be mediated by TCP of spin 0 or any integer.

\section{The details of $T_{F}$ (micro) are as follows:}

$$
\begin{aligned}
& T_{F}(\text { micro }) \cong 10^{5} S N F \cong 10^{44} \mathrm{GF} \cong 52.94 \text { dyne } \\
& 33.04 \times 10^{12} \mathrm{eV} \cong 33.04 \times 10^{3} \mathrm{GeV} \cong
\end{aligned}
$$

Strongest interaction (at $D_{i}=10^{-18} \mathrm{~cm}$, the theoretically calculated radius of a TCP or TRP within a nucleon) where $T_{F}($ micro $)=$ Thought force in microcosm.

As per Weinberg [11], there would have to remain a new class of "extra strong" interactions involving a few hundred $\mathrm{GeV}$. This "extra strong" interaction is the $\mathrm{T}_{\mathrm{F}}$ (micro) $\cong 33.04 \times 10^{3} \mathrm{GeV} \cong 33.04 \times 10^{12} \mathrm{eV} \cong$ "Technicolor" as indicated by Quigg [13].

Thus the existence of the "extra strong" interaction, indicated by Weinberg [11], often called "Technicolor" as pointed out by Quigg [13] is the $\mathrm{T}_{\mathrm{F}}$ (micro) in the microcosm. And this $\mathrm{T}_{\mathrm{F}}$ (micro) confirms the existence of TCP in the inherent presence of TRP in the microcosm.

\section{3. $T_{F}($ Macro $)=$ Thought Force in Macrocosm}

The existence of a "weakest force" which is much weaker even than the gravity has been indicated by Perkins [12] who pointed out, "The difficulty with an absolute conservation law for baryons is that, in analogy with charge conservation (linked to the existence of the electromagnetic field), there should be a new long-range field coupled to baryons, which should result in a difference in the apparent gravitational force on bodies with the same inertial mass but different baryon number. The Eotvos experiments indicate no such effects, and hence such a field would have to be very much weaker than gravity".

$\mathrm{T}_{\mathrm{F}}$ (macro) is theoretically found to be a new class of 'long-range weakest' force which is weaker even than the
GF. It is found that $\mathrm{T}_{\mathrm{F}}$ (macro) $\cong 10^{-3} G F$. This interaction is thought to be mediated by TCP of spin 0 or any integer.

The details of TF (macro) are as follows:

$$
\begin{aligned}
& T_{F}(\text { macro }) \cong 10^{-3} \mathrm{GF} \cong 10^{-42} \mathrm{SNF} \\
& \cong 2.958 \times 10^{-45} d y n e \cong 1.846 \times 10^{-33} \mathrm{eV} \\
& \cong 1.846 \times 10^{-42} \mathrm{GeV} \cong
\end{aligned}
$$

Long-range weakest interaction [at $D_{i}$ (Interacting

distance) $=$ radius of the universe $\left.\approx 1.482 \times 10^{29} \mathrm{~cm}\right]$

where $T_{F}$ (macro) $=$ Thought force in macrocosm.

The existence of the "weakest force" which is much weaker even than the gravity as explained by Perkins [12] and indicated by Weinberg [11] is detected to be the $T_{F}$ (macro). And this $\mathrm{T}_{\mathrm{F}}$ (macro) confirms the existence of TCP in the inherent presence of TRP into the quantum geometry of the universe.

\section{Quantum Fields Signify the Existence of $T_{F}$ (Micro) and $T_{F}$ (Macro)}

Thought force $\left(\mathrm{T}_{\mathrm{F}}\right)$ is bridging the microcosm and macrocosm through the generation of a universal web of quantum fields of $\mathrm{T}_{\mathrm{F}}$ (micro) and $\mathrm{T}_{\mathrm{F}}$ (macro).

Wilczek [17] expressed that intangible quantum fields fill everything in the universe, including the voids inside atoms and the universe, space between galaxies. Quantum electrodynamics has shown that even the vacuum of space, utterly devoid of matter or conventional forms of energy, actually seethes with activity. The possibility of the existence of a universal web of quantum fields signifies the existence of the postulated $\mathrm{T}_{\mathrm{F}}$ (micro) and $\mathrm{T}_{\mathrm{F}}$ (macro) due to the existence of the quantum mechanical activities of these TCP and TRP.

\section{Relationship between Thought Force $\left(T_{F}\right)$ and Universal Consciousness}

Pal et al [5] showed that the quantized energy $\left(\varepsilon_{T}\right)$ of TCP is responsible to cause the universal consciousness as well as the cosmic microwave background radiation (CMBR) temperature. Pal et al [5] showed further that the CMBR temperature exists due to the existence of $\boldsymbol{\varepsilon}_{T}$ of TCP radiated from the radiant mass of the universe. The existence of CMBR temperature confirms the existence of TCP in the inherent presence of TRP. The existence of TCP confirms the existence of thought force $\left(\mathrm{T}_{\mathrm{F}}\right)$. The same $\varepsilon_{T}$ is the energy responsible for generating thought force $\left(\mathrm{T}_{\mathrm{F}}\right)$. This $\mathrm{T}_{\mathrm{F}}$ exerts its functions both in vitro and in vivo (see Scheme-II). 


\section{Testability for the Existence of TCP and TRP Along with the Thought Force $\left(T_{F}\right)$}

It is fair to say that scientists have not yet detected the graviton whose existence is taken for granted for gravitational field. However, we propose here to verify the existence of these TCP and TRP along with the Thought force $\left(\mathrm{T}_{\mathrm{F}}\right)$ by different ways.

Nuclear physicists designed RHIC (Relativistic Heavy Ion Collider) at Brookhaven National Laboratory in Upton, New York and collided pairs of gold nuclei at high energies and observed the particles that sprayed from the impact point. The higher-energy results from $\mathrm{RHIC}$ for $\mathrm{Au}+\mathrm{Au}$ collisions at $130 \mathrm{GeV}$ (red) show greatly reduced yields, suggesting that the hot dense nuclear matter formed in these collisions is much more opaque than 'normal' nuclear matter as indicated by Adcox [18].

Broad [19] stated, "It is the quark-gluon plasma that physicists here are trying to make and study. Using the 2.4mile length of RHIC, they accelerate the nuclei of gold atoms (with 79 protons, 118 neutrons and no electrons, making them positively charged ions) to nearly the speed of light and then smash them together. The miniature fireball, up to 10,000 times as hot as the sun, briefly liberates the quarks and gluons -- in theory, at least. These two ingredients then quickly regroup and produce showers of thousands of particles that the physicists study. Immensely proud, scientists here say it is the first instrument ever made that uses head-on collisions of such high energy, letting the machine probe matter deeply.

"We want to understand the way the universe works, why a proton is a proton," said Dr. Wit Busza, a physicist at the Massachusetts Institute of Technology who helped design Phobos, one of RHIC's detectors. "We want to see the properties of this matter," he added. "Why is it so opaque? Why does it behave as it does? By answering all these questions, we'll be able to better understand the original state of the universe." They hope.

Along with hints of success, the physicists are also confronting a number of riddles. For instance, some particle jets are emerging in ways that defy expectations. Dr. Steven L. Manly, a physicist at the University of Rochester who works on the Phobos detector, likened them to pieces of a puzzle. "We're trying to figure out how this new picture fits together," he said. "It may be that we have an actual clue that something fundamental is different, something we just don't understand -- yet."

While most particles fly out with little energy -- hindered, it seems, by the dense plasma -- others have more than theory predicts. "The created matter is very opaque, very dense," said Dr. Busza of M.I.T. "Also, something new seems to be happening. I'm not sure what. Nature is telling us that there is some underlying simplicity here that we don't understand at the moment."

Stunning images show that some tracks are more curved than others, indicating that the particles had less momentum and a harder time breaking through the hot, dense medium.
Dr. Thomas Ludlam, a Star physicist, said some of the puzzles might turn out to be based in phenomena as simple as the expansion of the plasma "faster than we thought."

Like blind people trying to imagine the beauty of a fireworks display, the scientists are using their detectors to slowly build up an accurate picture of the tiny fireballs.

"It's no longer the terra incognita that we started with," Dr. Ludlam said. "We're starting to understand the landscape, at least a little bit." The ultimate goal is to produce evidence persuasive enough to lay unambiguous claim to the quark-gluon prize. Others have tried. In 2000, after years of effort, CERN, the leading European particlephysics laboratory presented circumstantial evidence that it had produced the rare form of matter. "But they stopped short of announcing a real discovery," said Dr. Ludlam, the Star physicist.

The implications are vast. A deep understanding of the plasma will not only help scientists grasp how the universe formed billions of years ago but will shed light on the forces and particles that rule nature today. "Ninety-nine point nine percent of matter on earth consists of quarks and gluons," Dr. Busza noted. Dr. Kirk of Brookhaven said the investigation was fundamental to the progress of science. "This universe came from somewhere," he said. "Was there a beginning and will there be an end? These are the most profound questions you can ask and are the kinds of things we're trying to investigate."

A Mysterious Force Gets a New Look. Scientists at Brookhaven National Laboratory are using the Relativistic Heavy Ion Collider to illuminate the workings of one of the four basic forces of nature.

Most physicists believe that a single super-force dominated the first instants of creation. But as the universe cooled and expanded after the Big Bang, the single force broke into the four observed today: gravity and electromagnetism, which act over long distances, and the weak and strong nuclear forces, which work inside the atom.

Physicists are using RHIC to study the strong force. Gluons are its carrier particle and quarks the object of its desire. The theory describing the strong force, quantum chromodynamics, contains some of physic's most daunting equations. Physicists hope that RHIC can test the theoretical assumptions and help simplify the picture.

Broad [19] further stated: The strong force is so fundamental, and its details so elusive, said Dr. Steven L. Manly of the University of Rochester, "that anything we can learn about it is important."

Physicists at the European Centre for Nuclear Research (CERN) research centre are trying to achieve high-power collisions of sub-atomic particles in their attempt to create mini-versions of the Big Bang that led to the birth of the universe 13.7 billion years ago.

The experiment at the CERN using Large Hadron Collider (LHC), creating a record for the energy of particle conditions, will allow researchers to examine the nature of matter and the origin of stars and planets.

Studying the data from millions of these high-energy collisions, RHIC scientists will be able to get not only a 
definite evidence of quark-gluon plasma (QGP) formation but also the existence of a Mysterious Force. Most physicists believe that a single super-force dominated the first instants of creation. This mysterious super-force is the Thought force $\left(T_{F}\right)$ in our view. It is possible that the evolution of all the fundamental fields is originated from this super-force that is the Thought force $\left(\mathrm{T}_{\mathrm{F}}\right)$. The Thought force $\left(T_{F}\right)$, in turn, is an expression of the universal consciousness. Thought force $\left(\mathrm{T}_{\mathrm{F}}\right)$ is the primordial quantum field that, in turn, functions as the primary unified field.

It is interesting to note the following significant statements mentioned by Broad [19]: Dr. Steven L. Manly said, "It may be that we have an actual clue that something fundamental is different, something we just don't understand -- yet." "The created matter is very opaque, very dense," said Dr. Busza of M.I.T. "Also, something new seems to be happening. I'm not sure what. Nature is telling us that there is some underlying simplicity here that we don't understand at the moment." All these statements through the experimental observations signify the possible existence of TCP and TRP that, in our view, are the ultimate constituents of matter and mind in the presence of thought force $\left(\mathrm{T}_{\mathrm{F}}\right)$ in vitro and thought force $\left(\mathrm{T}_{\mathrm{F}}\right)$ in vivo.

Scientists would detect the 'origin' of the QGP that might indicate the existence of these TCP and TRP. The researchers, in this way, through the utilization of 'nanotechnology', 'femtochemistry' and other sophisticated technologies may ultimately find out the existence and role of postulated TCP and TRP which are thought to be originated from the eternal void at the Big Bang to form the UM.

Further, transformation of energy waves into consciousness require an intermediate material stage, probably in the model of energy to matter and then from matter to the onset of consciousness in biological systems. The brain as a biological system converts the energy waves into chemical energy in the material form through the highly electrolytic neuro-chemicals. These chemicals get ionized in various degrees depending upon the energy input, coming out as information wave of specific length and frequency of the TCP, and facilitating the transport of information to different segments of the brain for interpretation. Pal et al $[3,5]$ expressed that the quantized energy ( $\varepsilon_{T}$ ) of the TCP is $4.384 \times 10^{-16} \mathrm{erg}$. The electrical activities inside the brain during the processing of information can be detected through Positron Emission Tomography and functional Magnetic Resonance Imaging (fMRI) in a target to find out the presence and role of these TCP and TRP.

\section{Discussion and Conclusion}

\subsection{Discussion}

The consciousness model incorporating TCP, TRP and thought force $\left(\mathrm{T}_{\mathrm{F}}\right)$ in vitro and thought force $\left(\mathrm{T}_{\mathrm{F}}\right)$ in vivo can provide several plausible guidelines to tackle many present day scientific enigmas, although this concept is new to the scientific world.

Physicists determined that underlying quantum fields give birth to elementary particles. Thought force $\left(T_{F}\right)$ being the primordial quantum field gives birth to TRP that appears to be the origin of all the matter particles. TCP is the carrier of thought force $\left(\mathrm{T}_{\mathrm{F}}\right)$ that, in turn, appears to be the origin of all the fields. TCP thus appears to be the origin of all the field particles (see Scheme-I and Scheme-II).

Thought force $\left(\mathrm{T}_{\mathrm{F}}\right)$ being the primordial quantum field functions as the original single primary unified field that is not only the origin of all the four fundamental fields along with both the $\mathrm{T}_{\mathrm{F}}$ (micro) and $\mathrm{T}_{\mathrm{F}}$ (macro) but also the origin of Thought force $\left(\mathrm{T}_{\mathrm{F}}\right)$ in vivo (see Scheme-II).

All the characteristics of a TCP have not been determined and naturally we do not know the exact spin of a TCP but we conjectured that the possible spin of a TCP would be 0 or any integer. The TCP that mediates $T_{F}$ (macro) may have a different spin than the TCP that mediates $\mathrm{T}_{\mathrm{F}}$ (micro).

Thought force $\left(\mathrm{T}_{\mathrm{F}}\right)$ in vivo is a type of force that represents the biological 'thought' which is the action of mind. The thought force $\left(\mathrm{T}_{\mathrm{F}}\right)$ in vivo is demonstrated in numerous experiments in which thought has an effect on a physical process (often known as mind over matter). This biological 'thought' is a type of force that can cause movement. Controlling movement through thought alone is observed in several experiments conducted by Birbaumer [20], Donoghue et al [21-24], Hatsopoulos [25] and Miguel [26]. These experiments thus signify the existence of thought force $\left(\mathrm{T}_{\mathrm{F}}\right)$ in vivo.

Bhaumik [6] pointed out, "Sheldrake has advanced the notion of an "extended mind" that can "reach out" to influence things well beyond the brain's boundaries. Like Nobel physicist Brian Josephson, he is deeply interested in finding a scientific explanation of psi phenomenon such as telepathy. A convincing proof of such phenomenon will obviously demonstrate that consciousness is not merely confined within the skull; it is capable of biological nonlocality. Mind is entangled with matter as matter is entangled with mind."

This biological nonlocality is possible through the application and utilization of quantum mechanical activities of these TCP and TRP in the inherent presence of Thought force $\left(\mathrm{T}_{\mathrm{F}}\right)$ in vitro and Thought force $\left(\mathrm{T}_{\mathrm{F}}\right)$ in vivo [along with $\mathrm{T}_{\mathrm{F}}$ (micro) and $\mathrm{T}_{\mathrm{F}}$ (macro)].

Wackermann [27-31] and Thaheld [32-35] conducted research to show a biological quantum entanglement and nonlocality existing between the mind-brains of separated human subjects and also separated human neuronal stem cells. Thaheld [32-35] has also tried to conduct research in the field of consciousness to unify physics and biology.

All these experiments conducted by Wackermann [27-31] and Thaheld 32-35] signify the existence of quantum mechanical activities of TCP and TRP, the ultimate constituents of matter and mind in the inherent presence of thought force $\left(\mathrm{T}_{\mathrm{F}}\right)$ in vitro and the thought force $\left(\mathrm{T}_{\mathrm{F}}\right)$ in vivo as indicated by Pal et al [5].

Wolf [36-42] has published a number of books to express the role of mind and consciousness through the quantum 
physics in order to interpret several aspects like Spirit, Soul, Matter, Self, A Mind-Expanding Journey Into the Realm Where Psyche and Physics Meet (1994), Mind into Matter, Matter Into Feeling, The Yoga of Time Travel: How the Mind Can Defeat Time (2004) etc. All these aspects can be explained through the existence of quantum mechanical activities of these TCP and TRP, the ultimate constituents of matter and mind in the inherent presence of thought force $\left(\mathrm{T}_{\mathrm{F}}\right)$ in vitro and the thought force $\left(\mathrm{T}_{\mathrm{F}}\right)$ in vivo as indicated by Pal et al [5].

Stapp's [43] work concerns the implications of quantum mechanics for consciousness. Stapp favors the idea that quantum waves collapse only when they interact with consciousness. He argues that quantum waves collapse when intelligent brains select one among the alternative quantum possibilities. His theory of how mind may interact with matter via quantum processes in the brain differs from that of Penrose and Hameroff. While the latter postulates quantum computing in the microtubules in brain neurons, Stapp postulates more global collapse via his 'mind like' wave-function collapse. His views are spelled out most clearly in his book, Mindful Universe: Quantum Mechanics and the Participating Observer. The 'mind like' wavefunction can be explained through the existence of quantum mechanical activities of these TCP and TRP, the ultimate constituents of matter and mind in the inherent presence of thought force $\left(\mathrm{T}_{\mathrm{F}}\right)$ in vitro and the thought force $\left(\mathrm{T}_{\mathrm{F}}\right)$ in vivo as indicated by Pal et al [5].

Pal et al [5] and $\mathrm{Pa}$ [44] showed that the quantized energy $\left(\varepsilon_{T}\right)$ of TCP represents universal consciousness. The individual consciousness owes its origin to the universal consciousness created by the same $\varepsilon_{T}$. This universal consciousness functions as a typical form of energy like light. Consciousness may thus be defined as a mental light through which a blind person can recognize any object through the use of his / her mental light.

The possible existence of the functional paranormal power like the Extra Sensory Perception (ESP) and Psychokinesis (PK) significantly indicates the existence of these TCP and TRP, the ultimate constituents of matter and mind in the inherent presence of thought force $\left(\mathrm{T}_{\mathrm{F}}\right)$ in vitro and the thought force $\left(\mathrm{T}_{\mathrm{F}}\right)$ in vivo as indicated by Pal et al [5]. These TCP, TRP and the thought force $\left(\mathrm{T}_{\mathrm{F}}\right)$ in vitro and thought force $\left(\mathrm{T}_{\mathrm{F}}\right)$ in vivo can exert their roles mentally (internally) as well as physically (externally).

\subsection{Conclusion}

The possible existence and functions of TCP and TRP in the inherent presence of thought force $\left(\mathrm{T}_{\mathrm{F}}\right)$ in vitro and the thought force $\left(\mathrm{T}_{\mathrm{F}}\right)$ in vivo are expressed here.

It appears that the standard model of physics is to be correctly tuned by ascertaining the constituents of quarks and leptons in the presence of unified field in order to form a possible gross bridge between mind and matter, present physics and cognitive science, psychology and natural sciences, classical physics and quantum physics.

\section{References}

[1] R. Penrose, Shadows of the Mind: A Search for the Missing Science of Consciousness (Oxford University Press) (1994)

[2] W. Van De Bogart, Earth portals: Exploring New Metaphors of Consciousness 1993 willard@earthportals.com

[3] D. Pal and A.U. De, Physics of consciousness and its model may provide guidelines to solve many scientific problems. Neuroquantology 1: 17-28 (2004)

[4] D. Pal, and A.U. De, Consciousness model: Significance of thought-carrying particles and thought-retaining particles in quantum measurement as well as cognitive problem. NeuroQuantology 2: 115-116 (2005)

[5] D. Pal, and A.U. De, The cosmic microwave background radiation temperature signifying the existence of the thought-carrying particle, thought retaining particle and thought force. NeuroQuantology 10: Issue3; 428-442 (September 2012)

[6] M. Bhaumik, Code Name GOD. (Penguin Books India Pvt. Ltd., 11 Community Centre, Panchsheel Park, New Delhi 110017 , India) pp. 132-133; 161-162; 167; 171; 177; 185$186 ; 89-190 ; 198(2006)$

[7] S. W. Hawking, A Brief History of Time from the Big Bang to Black Holes. (Bantam Books, 666 fifth Avenue, New York 10103), pp. 165; 136 (1989)

[8] Swami Ranganathananda, Science and Religion. (Advaita Ashrama, 5, Dehi Entally Road, Cal- 70001), pp. 130 (1988)

[9] F. Capra, The Tao of Physics. (Flemingo, Fontana paperbacks, the Collins Publishing group, 8 Grafton Street, London WIX 3LA), pp. 246 (1990)

[10] V. F. Weisskopf, The Origin of the Universe, The World of Physics, (Simon and Schuster, 1230 Avenue of Americas, New York 10020) 3, pp. 10 (1987)

[11] S. Weinberg, Conceptual Foundations of the Unified Theory of Weak and Electromagnetic Interactions. The World of Physics, (Simon and Schuster, 1230 Avenue of Americas, New York 10020); 3: pp.164 and 165 (1987)

[12] D. H. Perkins, Introduction to High Energy Physics. Addition-Wesley Publishing Co., Massachusetts 01867, U.S.A. pp. 17 and 364 (1982)

[13] C. Quigg, Elementary Particles and Forces. The World of Physics, (Simon and Schuster, 1230 Avenue of Americas, New York 10020); 2: 884 - 891 (1987)

[14] P. Davies, How bio-friendly is the universe? International Journal of Astrobiolog 2: 115 (2003)

[15] J.D.Barrow, and F .J. Tipler, The Anthropic Cosmological Principle 318 (1986)

[16] M. Gell-Mann, G. Chew, and A. Rosenfeld, Strongly Interacting Particles. The World of Physics, (Simon and Schuster, 1230 Avenue of Americas, New York 10020), 2: 797 (1987)

[17] F. Wilczek, Mass Without Mass I: Most of matter. Physics Today 11 (November, 1999)

[18] K. Adcox, PHENIX Collaboration. Phys Rev Lett 88: 022301 (2001) 
[19] W.J. Broad, In a Lab on Long Island, a Visit to the Big Bang. New York Times Articles Science. (January 14, 2003)

[20] N. Birbaumer, N. Ghanayim, T. Hinterberger, I. Iversen, B. Kotchoubey, A. Kübler, J. Perelmouter, E. Taub, and H. Flor, A spelling device for the paralysed. Nature 398, Number 6725; 297-9825 (March 1999)

[21] J. P. Donoghue, L. R. Hochberg, M. D. Serruya, G. M. Friehs, J. A. Mukand, M. Saleh, A. H. Caplan, A. Branner, D. Chen, and R. D. Penn, Neuronal ensemble control of Prosthetic Devices by a Human with tetraplegia. Nature 422, 13: 164-171 (July 2000)

[22] J. P. Donoghue, Connecting cortex to machines: recent advances in brain Interfaces, Nature Neuroscience Supplement. 5 1085-8 (November 2002)

[23] J. P. Donoghue, D. Mijail, G. N. Serruya, L. P. Hatsopoulos, F. R. Matthew, Brain-machine interface: Instant neural control of a movement signal. Nature 416, 14 Number 6877 , 141 (March 2002)

[24] J. P. Donoghue, R. K. Aaron, H. M. Herr, D. McK Ciombor, L. R. Hochberg, C. L. Briant, J. R. Morgan, and M. G Ehrlich, Horizons in prosthesis development for restoration of Limb Function. Am Accad Ortho Surg September. 14 (10) S198-S204 (2006)

[25] M. G. Hatsopoulos, D. Rubino, and K. A. Robbins, Propagating waves Mediate information transfer in the motor cortex. Nature Neuroscience. 9: 1549-1557 (2006)

[26] Nicolelis MAL Miguel, Brain-Machine interfaces to restore motor function and probe neural circuits. Nature Reviews Neuroscience. 4: 417-422 (2003)

[27] J. Wackermann, Towards a quantitative characterisation of functional states of the brain: From the non-linear methodology to the global linear description. International Journal of Psychophysiology. 34: 65-80 (1999)

[28] J. Wackermann, Characterization of states of consciousness based on global descriptors of brain electrical dynamics. Journal of Parapsychology. 63: 149-168 (1999)

[29] J. Wackermann, Benthin R, P"utz P. An ESP experiment with the Sequential Card Search Task. Proc 44th Ann Conv Parapsychol Assoc, New York, NY; 334-348 (2001)

[30] J. Wackermann, Putz P, Buchi S, Strauch I, Lehmann D. Brain electrical activity and subjective experience during altered states of consciousness: ganzfeld and hypnagogic states. International Journal of Psychophysiology 46: 123$146(2002)$
[31] J. Wackermann, C. Seiter, H. Keibel and H. Walach. Correlations between brain electrical activities of spatially separated human subjects. Neuroscience Letters 336: 60-64 (2003)

[32] F. H. Thaheld, Biological nonlocality and the mind-brain interaction problem: comments on a new empirical approach Biosystems 70:35-41 (2003)

[33] F. H. Thaheld, A method to explore the possibility of nonlocal correlations between brain Electrical activities of two spatially separated animal subjects. Biosystems 73 (3):205-16 (2004)

[34] F. H. Thaheld, An interdisciplinary approach to certain fundamental issues in the fields of physics and biology: towards a unified theory. Biosystems 80:41-56 (2005)

[35] F. H. Thaheld, Does consciousness really collapse the wave function? A possible objective biophysical resolution of the measurement problem. Biosystems 81(2):113-24 (2005)

[36] F. A. Wolf, The Body Quantum: The New Physics of Body, Mind and Health. (Macmillan Pub Co.) (1986)

[37] F. A. Wolf, Star Wave: Mind, Consciousness and Quantum Physics. (Harper Perennial) (1989)

[38] F. A. Wolf, The Dreaming Universe: A Mind-Expanding Journey Into the Realm Where Psyche and Physics Meet. (Simon \& Schuster) (1994)

[39] F. A. Wolf, The Spiritual Universe: One Physicists Vision of Spirit, Soul, Matter, and Self. (Published by Simon \& Schuster, Moment Point Press) (1998)

[40] F. A. Wolf, Mind into Matter: A New Alchemy of Science and Spirit. (Moment Point Press) (2000)

[41] F. A. Wolf, Matter Into Feeling: A New Alchemy of Science and Spirit. (Moment Point Press) (2002)

[42] F. A. Wolf, The Yoga of Time Travel: How the Mind Can Defeat Time. (Quest Books) (2004)

[43] H.P. Stapp, Mindful Universe: Quantum Mechanics and the Participating Observer. (Springer) (2007)

[44] D. Pal, Existence of universal consciousness and its characteristics. Accepted for publication in 2014 International Conference on Advanced Education and Management (ICAEM2014) Beijing, China (2013) 